
\title{
$\begin{array}{ll}\text { Research Square } & \begin{array}{l}\text { Preprints are preliminary reports that have not undergone peer review. } \\ \text { They should not be considered conclusive, used to inform clinical practice, } \\ \text { or referenced by the media as validated information. }\end{array}\end{array}$
}

\section{Text Data Augmentation for Deep Learning}

Connor Shorten ( $\sim$ cshorten2015@fau.edu )

Florida Atlantic University https://orcid.org/0000-0001-6253-6861

\section{Taghi M. Khoshgoftaar}

Florida Atlantic University

\section{Borko Furht}

Florida Atlantic University

\section{Research Article}

Keywords: Data Augmentation, Natural Language Processing, Overfitting, Big Data, NLP, Text Data

Posted Date: June 29th, 2021

DOl: https://doi.org/10.21203/rs.3.rs-650804/v1

License: (c) (1) This work is licensed under a Creative Commons Attribution 4.0 International License. Read Full License

Version of Record: A version of this preprint was published at Journal of Big Data on July 19th, 2021. See the published version at https://doi.org/10.1186/s40537-021-00492-0. 


\section{Abstract}

Natural Language Processing (NLP) is one of the most captivating applications of Deep Learning. In this survey, we consider how the Data Augmentation training strategy can aid in its development. We begin with the major motifs of Data Augmentation summarized into strengthening local decision boundaries, brute force training, causality and counterfactual examples, and the distinction between meaning and form. We follow these motifs with a concrete list of augmentation frameworks that have been developed for text data. Deep Learning generally struggles with the measurement of generalization and characterization of overfitting. We highlight studies that cover how augmentations can construct test sets for generalization. NLP is at an early stage in applying Data Augmentation compared to Computer Vision. We highlight the key differences and promising ideas that have yet to be tested in NLP. For the sake of practical implementation, we describe tools that facilitate Data Augmentation such as the use of consistency regularization, controllers, and offline and online augmentation pipelines, to preview a few. Finally, we discuss interesting topics around Data Augmentation in NLP such as task-specific augmentations, the use of prior knowledge in self-supervised learning versus Data Augmentation, intersections with transfer and multi-task learning, and ideas for Al-GAs (Al-Generating Algorithms). We hope this paper inspires further research interest in Text Data Augmentation.

\section{Full Text}

This preprint is available for download as a PDF. 